\title{
Adult Food Allergy Prevalence: Reducing Questionnaire Bias
}

\author{
Letícia A. Silva Anna Flávia M. Silva Âmela C. Ribeiro Augusto O. Silva \\ Felipe A. Vieira Gesmar R.S. Segundo
}

Allergy and Immunology Division, Pediatrics Department, Universidade Federal de Uberlandia, Uberlandia, Brazil

\section{Keywords}

Food allergy · Prevalence $\cdot$ Epidemiology $\cdot$ Allergy

\begin{abstract}
Background: Food allergy (FA) prevalence has increased in the last decades, but epidemiologic studies could show overestimated results. The objective of this study is to estimate the prevalence of immediate FA in adults in a region of Central Brazil, using a questionnaire to try to reduce misperceptions about FA reaction. Methods: A cross-sectional study was conducted, enrolling an adult population aged 18-65 years comprised of families in a Central Brazilian city. In the first phase, participants answered a self-administered questionnaire for FA screening. In the second phase, the participants who reported an FA in the first questionnaire were visited to complete the second questionnaire applied by trained researchers. Results: Of the 4,916 adults visited, 1,583 returned a completed questionnaire. Reported FA occurred in 171 (10.8\%) subjects, and the more frequent citations were cow's milk, pork, fruits, shrimp, and vegetables. One hundred and four of these individuals completed the second questionnaire, and $15(1.0 \%)$ were considered to have an FA diagnosis. The main foods were fruits, followed by cow's milk, shrimp, pork, and vegetables. Conclusion: After use of a specific questionnaire to recognize possible lgE-
\end{abstract}

mediated FAs, a low frequency of FA was considered in this population. Use of a directed questionnaire administered by trained researchers could be an alternative for epidemiological IgE-mediated FA studies to achieve more accurate results.

(c) 2017 S. Karger AG, Basel

\section{Introduction}

Food allergy (FA) is an adverse immune response that occurs reproducibly upon contact with a specified food. FA frequency has increased by $18 \%$ in the last 10 years, but prevalence studies based on self-reported FA show a high familiar and individual perception of FA $[1,2]$. In this context, the objective of this study is to estimate the prevalence of immediate FA in adults in a region of Central Brazil, using a questionnaire to try to reduce misperceptions about FA reaction.

\section{Methods}

A cross-sectional study was conducted during the period of December 2014 to July 2015 in an enrolled adult population aged 18-65 years, comprised of families in a Family Health Strategy (FHS) area responsible for primary care in the St. George II and III

\section{KARGER}

(c) 2017 S. Karger AG, Basel

E-Mail karger@karger.com

www.karger.com/iaa
Correspondence to: Dr. Gesmar Rodrigues Silva Segundo

Pediatrics Department, Faculty of Medicine

Universidade Federal de Uberlandia - Campus Umuarama, Bloco $2 \mathrm{H}$

Uberlandia, MG 38400-920 (Brazil)

E-Mail gesmar@famed.ufu.br 
Fig. 1. Study algorithm flowchart. FA, food allergy.

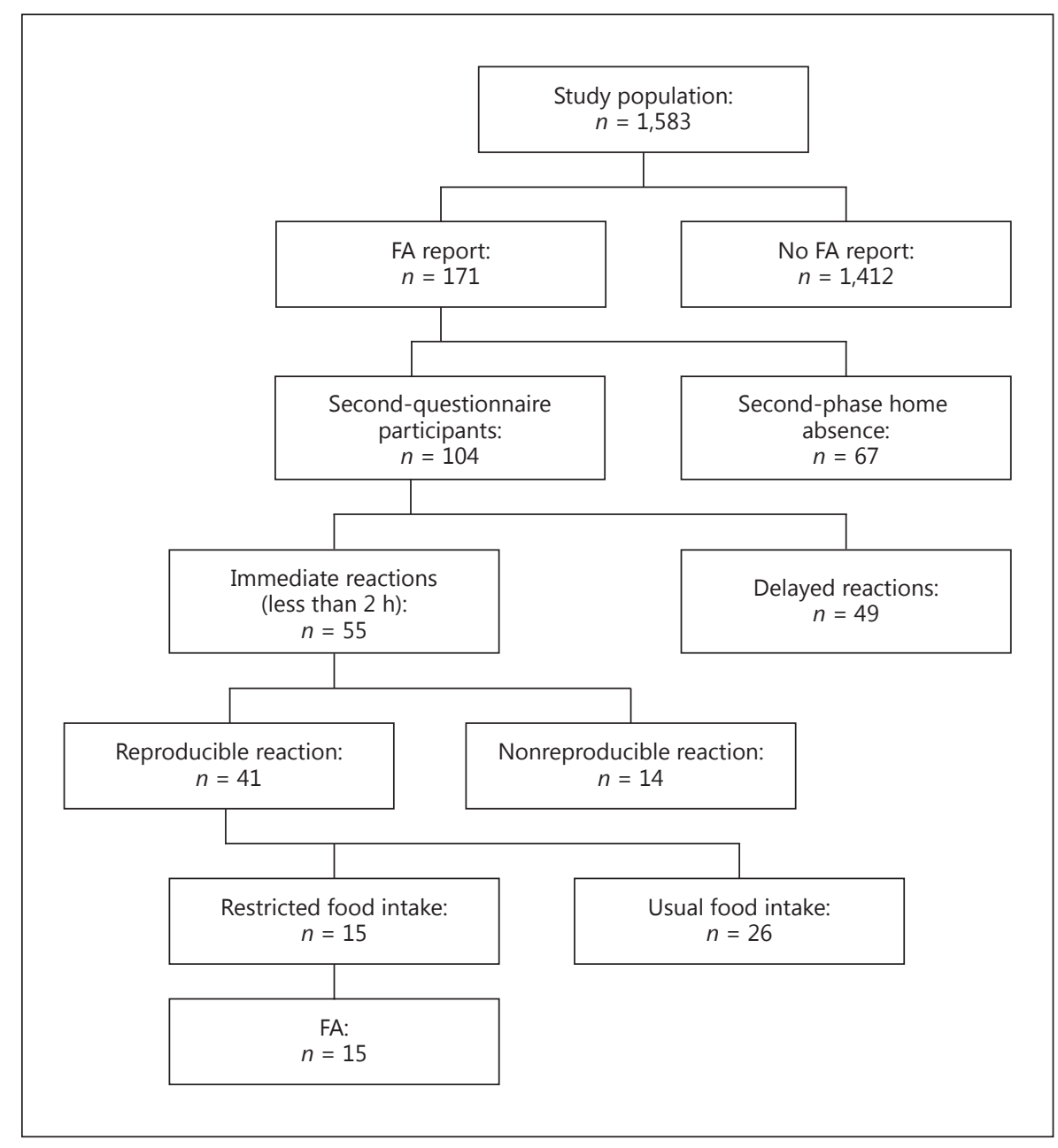

district in Uberlandia, a Central Brazilian city. All participants were informed about this study, and signed consent was obtained. The exclusion criteria were: illiteracy, refusal to participate, refusal to sign the consent form, absence from home, or incorrect filling in of the questionnaire. This study was approved by the Ethics Committee of the Federal University of Uberlandia.

This study was performed in 2 phases, as shown in Figure 1. In the first phase, a validated self-administered questionnaire for FA screening (online suppl. Table S1; for all online suppl. material, see www.karger.com/doi/10.1159/000453036) was used to collect the data [3]. The FHS mapped 4,916 adults living in the area in 2,325 houses during the study period. To reach the target population, the researchers accompanied the FHS agents on home visits in the district. For each house, the researchers made at least 3 attempts on different days and at different times before considering exclusion due to absence from home. The first questionnaire was delivered to homes where at least 1 adult was present to receive the researchers and the study explanation. A total of 231 (4.7\%) adults were illiterate and therefore excluded. We did not receive permission to visit a condominium with 176 apartments with approximately 250 adults. There was no one present during the 3 attempts in around 500 homes, where we were not able to explain or deliver the ques- tionnaires. In the end, the researchers delivered around 3,350 questionnaires.

The data collected was about gender, age, any atopy (history of rhinitis, atopic dermatitis, and asthma), and reported FA and allergic symptoms related to food, including the type of food and the symptoms experienced.

In the second phase, the participants who reported an FA in the first questionnaire were visited to complete the second questionnaire, applied by the trained researchers. This questionnaire provided more detailed questions about the food involved in the FA reaction, associated symptoms, the time between food intake and the onset of the FA reaction, the reproducibility of the symptoms, and food exclusion in the daily diet (online suppl. Table S2) as previously described $[4,5]$. These questions were geared toward building up a clinical history, and a possible diagnosis of FA was considered if the replies met all of the following 3 criteria: the reaction symptoms started within $2 \mathrm{~h}$ of ingestion of the food implicated, the reaction was reproducible, and the food was excluded from the daily diet.

Fisher's exact test and the $\chi^{2}$ test were used for categorical variables. The level of significance for all statistical tests was 2-sided $(p<0.05)$. All analyses were conducted using GraphPad Prism 5.0 (La Jolla, CA, USA). 
Table 1. Characteristics reported in the general adult FA questionnaire

\begin{tabular}{lcc}
\hline Characteristic & Reported FA & Nonreported FA \\
\hline Subjects & $171(10.8)$ & $1,412(89.2)$ \\
Gender & $36(21.0)$ & $510(36.1)$ \\
Male & $134(78.9)$ & $875(63.89)$ \\
Female & & \\
Reported allergic diseases & $17(9.9)$ & $53(3.7)$ \\
Asthma & $41(24.0)$ & $182(12.9)$ \\
Allergic rhinitis & $19(11.1)$ & $23(1.3)$ \\
Atopic dermatitis & & \\
Main causative food reaction ${ }^{1}$ & $35(2.2)$ & \\
Cow's milk & $34(2.1)$ & \\
Pork & $26(1.6)$ & \\
Fruits & $21(1.3)$ & \\
Shrimp and other seafood & $15(0.9)$ & \\
Vegetables & $11(0.7)$ & \\
Fish & $6(0.4)$ & \\
Egg & $84(49.1)$ & \\
Main symptoms associated with food reactions & \\
Red spots on the skin & $44(25.7)$ & \\
Abdominal pain & & \\
Swelling of the mouth and/ & & \\
$\quad$ or eyes & $38(22.2)$ & \\
Diarrhea & $37(21.6)$ & \\
Dyspnea & $24(14.0)$ & $(3.5)$ \\
Runny nose & & \\
Itchiness & & \\
Others symptoms & & \\
\hline
\end{tabular}

Values are presented as numbers (\%). The total number of subjects was 1,583 . FA, food allergy. ${ }^{1}$ In relation to 1,583 participants. ${ }^{2}$ In relation to 171 reported FA.

\section{Results}

The FHS unit received 1,583 completed questionnaires $(32.2 \%$ of 4,916$)$ and signed consent forms. There was a significant female gender predominance (63.7\%) in these returned items. Reported FA occurred in 171 (10.8\%) first questionnaires. The foods implicated in the FA, the symptoms associated with a food reaction, and any other types of allergic diseases are described in $\mathrm{Ta}$ ble 1. Several other foods were cited in the surveys, each one in small amounts. An FA reaction to 1 food was cited $106(62.0 \%)$ times; 26 (15.2\%) subjects reported reactions to 2 foods, 17 (9.9\%) mentioned reactions to 3 foods, and 5 (5.3\%) cited reactions to 4 or more foods.

In the second phase, all individuals who reported an FA history were revisited to answer a specific survey about IgE-mediated FAs. However, 67 people were unavailable at the second visit performed by researchers (af-
Table 2. Clinical responses after the specific FA questionnaire

\begin{tabular}{ll}
\hline Characteristic & Value \\
\hline Subjects, $n$ & 104 \\
Gender & \\
Male & $22(21.1)$ \\
Female & $82(78.8)$ \\
Causative food ${ }^{1}$ & \\
Fruit & $6(0.4)$ \\
Cow's milk & $4(0.3)$ \\
Shrimp and other seafood & $4(0.3)$ \\
Pork & $3(0.2)$ \\
Vegetables & $3(0.2)$ \\
Pepper & $1(0.1)$ \\
Corn & $1(0.1)$ \\
Gluten & $1(0.1)$ \\
\hline Main symptoms & \\
Abdominal pain & \\
Red spots on the skin & $8(53.3)$ \\
Pruritus & $6(40.0)$ \\
Nausea/vomiting & $6(40.0)$ \\
Eye swelling & $5(33.3)$ \\
Mouth swelling & $3(20.0)$ \\
Dyspnea & $2(13.3)$ \\
Diarrhea & $2(13.3)$ \\
Runny nose & $2(13.3)$ \\
\hline & $1(6.7)$ \\
\hline &
\end{tabular}

Values are presented as numbers (\%) unless otherwise stated. FA, food allergy. ${ }^{1}$ In relation to 1,516 participants. ${ }^{2}$ In relation to 15 reported FA. ter 3 attempts) and $104(60.8 \%)$ participants responded to the second questionnaire.

After the second questionnaire, only 15 (1.0\%) people were considered to have a probable IgE-mediated FA. The characteristics, the food implicated in the FA reactions, and the symptoms associated with FAs are described in Table 2. In that questionnaire, $6(0.4 \%)$ persons reported a fruit allergy that included watermelon $(n=3)$, pineapple $(n=1)$, avocado $(n=1)$, and tomato $(n=1)$, while vegetable allergies were all attributed to cucumber.

\section{Discussion}

The present study demonstrated an initial FA report in $10.80 \%$ of the adult individuals, similar to the rates described in adult FA prevalence studies (i.e., 7-35\%) [2, 6-9]. This huge variation probably occurred as a result of 
methodological, cultural, and food intake differences [69]. The increased female gender was perhaps due to the data collection format, consisting of home visits during business hours depending on the schedule of the FHS agents. On the other hand, previous studies observed a higher perception of FA in females, as seen in our data $[7,8]$.

After the second questionnaire, only 15 (14.2\%) of those with an initial FA report met the probable IgE-mediated FA criteria; FA reports declined from 10.8 to $1.0 \%$. The changes in FA prevalence were substantial (almost 10fold), especially for cow's milk (from 2.2 to $0.3 \%$ ), probably because of the confusion between lactose intolerance and FA in the first instance, and for pork (from 2.1 to $0.2 \%$ ), probably related to indigestion associated with pork meat cooking methods. Other foods such as fruit (from 1.6 to $0.4 \%$ ), shrimp and other seafood (from 1.3 to $0.3 \%$ ), and vegetables (from 0.9 to $0.2 \%$ ) also presented a slight reduction in allergy prevalence. The results of the second questionnaire are similar to the findings of FA prevalence studies performed using food challenges described in a recent published meta-analysis, and they are probably more assertive than previous studies using only prick tests, specific IgE, and a generic questionnaire only $[6,8,9]$.

Fruits and vegetables are considered the most frequent cause of FA after adolescence, in concordance with the present study, and they are associated with pollen food syndrome and lipid transfer protein syndrome [10]. The absence of a reported peanut allergy is noteworthy, compatible with the information previously published in another Brazilian study [3].

Only one study has used this methodology to assess FA before, but in childhood. In that study, they found a parental FA perception in $15.1 \%$ of the participants, and after specific questions the number decreased to $3.7 \%$ [5].
Differences related to food allergens and the presence of gastrointestinal manifestations in the present study were greater than those observed in the present study (around $80 \%$ ) compared to the Korean study (around 10\%), and this was probably related to age differences and different cultural habits.

This study has limitations, such as the nonresponse rate in the first phase, 67 (39.2\%) cases of home nonattendance among the selected participants in the second phase, and the fact that it was conducted in a city region, but these limitations do not invalidate the data. Another important point is related to those individuals who excluded a specific food or a group of foods after one isolated reaction; since they had no reproducible reaction in a repeated food intake, the diagnosis of FA by our questionnaire was not possible and could have decreased the estimated prevalence. However, even in the clinical approach, reproducibility is one of the major pillars of FA diagnosis.

In conclusion, after use of a specific questionnaire to recognize probable IgE-mediated FAs, a low frequency of FA was considered in this population. Use of a directed questionnaire administered by trained researchers could be an alternative for epidemiological IgE-mediated FA studies to achieve more accurate results.

\section{Acknowledgement}

This study was funded by the Young Talent Exchange Program for Science 2013 offered by the Coordenação de Aperfeiçoamento de Pessoal de Nível Superior (Capes) - Brazilian Agency.

\section{Disclosure Statement}

The authors declare that there are no conflicts of interest to disclose.

\section{References}

1 Branum AM, Lukacs SL: Food allergy among children in the United States. Pediatrics 2009; 124:1549-1555.

2 Sicherer SH: Epidemiology of food allergy. J Allergy Clin Immunol 2011;127:594-602.

3 Gonçalves LC, Guimarães TC, Silva RM, et al: Prevalence of food allergy in infants and preschoolers in Brazil. Allergol Immunopathol (Madr) 2016;44:497-503.

4 Lyra NR, Motta ME, Rocha LA, et al: Adverse reactions to foods and food allergy: development and reproducibility of a questionnaire for clinical diagnosis. J Allergy (Cairo) 2013; 2013:920679.
5 Park M, Kim D, Ahn K, Kim J, Han Y: Prevalence of immediate-type food allergy in early childhood in Seoul. Allergy Asthma Immunol Res 2014;6:131-136.

6 Rona RJ, Keil T, Summers C, et al: The prevalence of food allergy: a meta-analysis. J Allergy Clin Immunol 2007;120:638-646.

7 Vierk KA, Koehler KM, Fein SB, Street DA: Prevalence of self-reported food allergy in American adults and use of food labels. J Allergy Clin Immunol 2007;119:1504-1510.
8 Nwaru BI, Hickstein L, Panesar SS, Roberts G, Muraro A, Sheikh A: Prevalence of common food allergies in Europe: a systematic review and meta-analysis. Allergy 2014;69:9921007.

9 Soller L, Ben-Shoshan M, Harrington DW, et al: Adjusting for nonresponse bias corrects overestimates of food allergy prevalence. J Allergy Clin Immunol Pract 2015;3:291-293.

10 Fernández-Rivas M: Fruit and vegetable allergy. Chem Immunol Allergy 2015;101:162170 . 